\title{
GLP-2 Analogues as First Specific Treatment of Intestinal Failure
}

\author{
Irina Blumenstein \\ Department of Gastroenterology, Hepatology and Clinical Nutrition, University Hospital Frankfurt, \\ Frankfurt am Main, Germany
}

Keywords

Growth factors · Trophic hormones - Specific treatment . Intestinal failure $\cdot$ Teduglutide $\cdot$ Mode of action $\cdot$ Pivotal study data $\cdot$ Treatment response prediction $\cdot$ New substances in phase clinical trials

\section{Abstract}

Growth factors and other trophic hormones play an important role in the specific treatment of intestinal failure, with teduglutide being the first approved medication specifically for use in patients with intestinal failure in conjunction with specialized nutritional support. This article focusses on the mode of action, the pivotal study data, treatment response prediction as well as new substances in phase clinical trials.

(c) 2019 S. Karger AG, Basel

\section{Introduction}

Apart from a fully or partially parenteral nutritional (PN) therapy with substitution of water- and fat-soluble vitamins and trace elements, a pharmacological intervention in patients with chronic intestinal failure (IF) is additionally useful. Depending on the remaining length of the small and large intestine and their continuity, motility inhibitors, stool thickeners, pancreatic enzymes, proton pump inhibitors, and bile acid binders can be used to reduce fluid and electrolyte losses as well to improve intestinal passage time and stool frequency. Growth factors and other trophic hormones have been used to enhance intestinal adaptation [1]. Recombinant human growth hormone was the first medication approved by the US
FDA specifically for use in patients with short bowel syndrome (SBS) in conjunction with specialized nutritional support [2]. However, the use of growth hormone has been limited, largely owing to concerns with regard to efficacy and the fact that only short-term use was approved $[3,4]$.

\section{GLP-2 Analogue Teduglutide}

The recombinant analogue of human glucagon-like peptide-2 (GLP-2) teduglutide (Gattex_, Revestive_) is a novel therapeutic option for SBS. GLP-2 is a naturally occurring hormone regulating the growth, proliferation, and maintenance of cells in the gastrointestinal tract. Subcutaneous teduglutide is the first long-term medical therapy approved for the treatment of adult and pediatric patients with SBS who are dependent on parenteral support (PN and/or intravenous fluids).

\section{Efficacy}

In a pivotal, double-blind, multicenter, phase III study (STEPS) in adult patients with SBS who were dependent on parenteral support, a total of $54 \%$ of all patients had at least one additional infusion-free day a week as a result of daily therapy with teduglutide $0.05 \mathrm{mg} / \mathrm{kg} / \mathrm{day}$. A reduction of PN by more than $20 \%$, the primary endpoint of the study, was achieved by $46 \%$ after 20 weeks and by $63 \%$ of all patients after 24 weeks compared to 6 and $30 \%$ of all patients in the placebo arm [5]. The long-term data over a period of 30 months showed that a further reduction of

\section{KARGER}

(C) 2019 S. Karger AG, Basel 
infusion days is to be expected. After completion of the STEPS-2 study, the extension study of STEPS, 13/88 patients were completely weaned from PN. A clinical response to teduglutide (reduction of PN by $20 \%$ ) was observed in $93 \%$ of all patients treated with teduglutide [6].

\section{Effects of GLP-2 on Gastrointestinal Absorption}

Intestinotrophic effects of GLP-2 and its analogues are reduced gastric motility and secretion, improved intestinal barrier function, increased intestinal blood flow, and stimulation of gastrointestinal epithelium growth $[7,8]$. These effects lead to the positive results observed in the clinical studies.

\section{Safety}

The increase in intestinal absorption is a result of increased proliferation of the mucosa, which has shortterm side effects on the small intestine and long-term risks. Side effects include abdominal pain and bloating, respiratory infections, nausea and vomiting, and reactions at the injection site - as a short-lived proteohormone, teduglutide must be injected subcutaneously daily. Patients with stoma report more frequent complications at the artificial anus [5, 9-11].

The long-term risks concern obstructions in the intestine, bile ducts, and pancreatic duct. The formation of polyps is promoted in the large intestine. A colonoscopy with removal of any polyps is therefore performed before the start of treatment and at regular intervals thereafter [6]. A pharmacovigilance plan in Europe and a risk evaluation and mitigation strategy in the USA are ensuring that these precautionary measures are actually observed in a registry, in which all patients receiving teduglutide are entered after their consent. Up to date, no increased risk of cancer has been reported.

Results from a phase III trial in pediatric SBS patients with IF (SBS-IF) dependent on PN were consistent with those in adults, resulting in an approval for the treatment of pediatric SBS-IF patients as well [12].

\section{Prediction of Response}

Since the use of GLP-2 analogues leads to high costs in the medium to long term, the identification of predictors for a response to the therapy and its course is extremely important. In a real world study published by Schoeler and colleagues [13], there was a significant reduction in parenteral support after 24 and 48 weeks (by 11.0 and $36.6 \%$, respectively; $p<0.05$ ). Further major improve- ments were made in several patients after over 1 year (reduction by $79.3 \%, p<0.05)$. The proportion of patients who reduced parenteral support by at least $20 \%$ was 33.3 , 54.5 , and $71.3 \%$ after 24 weeks, 48 weeks, and beyond 1 year, respectively. Patients on daily parenteral support showed late but strong amelioration. The reduction of thirst was the earliest marker for response. While stool consistency increased $(p<0.01)$, stool frequency decreased $(p<0.05)$ significantly after 12 weeks. This reduction was even more pronounced in patients with colon in continuity, supported by a stronger physiological expression of the GLP-2 receptor in the colon than in the small intestine. Thus, stool consistency and frequency, as well as the sensation of thirst, were identified as early clinical markers of response in this large single-center clinical study. Clinical and molecular observations support the role of the colon as an important target organ of teduglutide [13].

\section{New Substances in Clinical Trials}

Glepaglutide, a novel long-acting GLP-2 analogue, for reducing fecal output and increasing intestinal absorption in patients with SBS has shown promising results in a phase II trial. Patients (aged $\geq 18$ to $\leq 90$ years) with SBS and with a fecal wet weight output of $1,500 \mathrm{~g} /$ day or more were randomly assigned to receive one of six dose sequences of glepaglutide (10, $1 \mathrm{mg} ; 10,0.1 \mathrm{mg} ; 1,10 \mathrm{mg} ; 1$, $0.1 \mathrm{mg} ; 0.1,10 \mathrm{mg}$; or $0.1,1 \mathrm{mg}$ ). Patients received daily subcutaneous injections of the first assigned dose of glepaglutide for 3 weeks, followed by a washout period of 4-8 weeks, and then the second dose of glepaglutide for 3 weeks. The primary endpoint was the absolute change from baseline in fecal wet weight output, measured separately over the two treatment periods. 18 patients were randomly assigned and treated with glepaglutide; 16 patients completed the trial. Treatment with 1 and $10 \mathrm{mg}$ glepaglutide changed the adjusted mean fecal output by $-592 \mathrm{~g} /$ day $(95 \% \mathrm{CI}-913$ to $-272 ; p=0.002)$ and $-833 \mathrm{~g} /$ day $(-1,152$ to $-515 ; p=0.0002)$ from baseline, respectively. No changes were observed with $0.1 \mathrm{mg}$ glepaglutide. Related or possibly related serious adverse events were reported in two patients in the 0.1-mg dose group and two patients in the 10-mg dose group. These events included abdominal pain, stoma obstruction, catheterrelated sepsis, and infection of unknown origin. No patients died during the trial. Glepaglutide was well tolerated in this study and was associated with improved intestinal absorption in patients with SBS with 1 and $10 \mathrm{mg}$ glepaglutide, but not with $0.1 \mathrm{mg}$ glepaglutide [14]. Larger phase III clinical trials of longer durations have been initiated to fully assess the safety and efficacy of glepaglutide. 
So far, the phase II/III studies of all GLP-2 analogues were and are performed in patients on stable parenteral support, often many years out from the last resection. It would be interesting to see the efficacy of these substances far earlier in the course of the disease, even shortly after intestinal resection. The outcome after the removal of small intestine and colon segments seems to be predictable, the early application of intestinal growth factors holds the potential of a possibly faster oral autonomy, which is not yet exploited.
GLP-2 analogues open up the possibility of a specific therapy of IF for the first time. Further studies are needed to evaluate the efficacy of an early postoperative use of these substances.

\section{Disclosure Statement}

The author did not provide a conflict of interest statement.

\section{References}

1 McMellen ME, Wakeman D, Longshore SW, McDuffie LA, Warner BW. Growth factors: possible roles for clinical management of the short bowel syndrome. Semin Pediatr Surg. 2010 Feb;19(1):35-43.

2 Buchman AL, Scolapio J, Fryer J. AGA technical review on short bowel syndrome and intestinal transplantation. Gastroenterology. 2003 Apr;124(4):1111-34.

3 Scolapio JS. Short bowel syndrome: recent clinical outcomes with growth hormone. Gastroenterology. 2006 Feb;130(2 Suppl 1):S1226.

4 Steiger E. Guidelines for pharmacotherapy, nutritional management, and weaning parenteral nutrition in adult patients with short bowel syndrome: introduction. J Clin Gastroenterol. 2006 May-Jun;40 Suppl 2:S73-4.

5 Jeppesen PB, Pertkiewicz M, Messing B, Iyer $\mathrm{K}$, Seidner DL, O'keefe SJ, et al. Teduglutide reduces need for parenteral support among patients with short bowel syndrome with intestinal failure. Gastroenterology. $2012 \mathrm{Dec}$ 143(6):1473-1481.e3.
6 Schwartz LK, O’Keefe SJ, Fujioka K, Gabe SM, Lamprecht G, Pape UF, et al. Long-Term Teduglutide for the Treatment of Patients With Intestinal Failure Associated With Short Bowel Syndrome. Clin Transl Gastroenterol. 2016 Feb;7(2):e142.

7 Cheeseman CI, Tsang R. The effect of GIP and glucagon-like peptides on intestinal basolateral membrane hexose transport. Am J Physiol. 1996 Sep;271(3 Pt 1):G477-82.

8 Jeppesen PB, Sanguinetti EL, Buchman A, Howard L, Scolapio JS, Ziegler TR, et al. Teduglutide (ALX-0600), a dipeptidyl peptidase IV resistant glucagon-like peptide 2 analogue, improves intestinal function in short bowel syndrome patients. Gut. 2005 Sep;54(9): 1224-31.

9 Ukleja A, To C, Alvarez A, Lara LF. LongTerm Therapy With Teduglutide in Parenteral Support-Dependent Patients With Short Bowel Syndrome: A Case Series. JPEN J Parenter Enteral Nutr. 2018 May;42(4):821-5.

10 Jeppesen PB, Gilroy R, Pertkiewicz M, Allard JP, Messing B, O’Keefe SJ. Randomised placebo-controlled trial of teduglutide in reducing parenteral nutrition and/or intravenous fluid requirements in patients with short bowel syndrome. Gut. 2011 Jul;60(7):902-14.
11 O’Keefe SJ, Jeppesen PB, Gilroy R, Pertkiewicz M, Allard JP, Messing B. Safety and efficacy of teduglutide after 52 weeks of treatment in patients with short bowel intestinal failure. Clin Gastroenterol Hepatol. 2013 Jul; 11(7):815-23.e1.

12 Carter BA, Cohran VC, Cole CR, Corkins MR, Dimmitt RA, Duggan C, et al. Outcomes from a 12-Week, Open-Label, Multicenter Clinical Trial of Teduglutide in Pediatric Short Bowel Syndrome. J Pediatr. 2017 Feb; 181:102-111.e5.

13 Schoeler M, Klag T, Wendler J, Bernhard S, Adolph M, Kirschniak A, et al. GLP-2 analog teduglutide significantly reduces need for parenteral nutrition and stool frequency in a real-life setting. Therap Adv Gastroenterol. 2018 Aug; 11:1756284818793343.

14 Naimi RM, Hvistendahl M, Enevoldsen LH, Madsen JL, Fuglsang S, Poulsen SS, et al. Glepaglutide, a novel long-acting glucagonlike peptide- 2 analogue, for patients with short bowel syndrome: a randomised phase 2 trial. Lancet Gastroenterol Hepatol. 2019 May;4(5):354-63. 